\title{
Probing New Physics in Charm Couplings with Kaon and Other Hadron Processes
}

\author{
Jusak Tandean*广 \\ Department of Physics, National Taiwan University, \\ Taipei 106, Taiwan \\ E-mail: jtandean@phys.ntu.edu.tw
}

\begin{abstract}
It is possible that the low-energy effects of physics beyond the standard model can be parametrized mainly by anomalous couplings of quarks to the $W$ boson. Such couplings can generate potentially significant contributions to various transitions that can be probed by current and future experiments. This work explores constraints on anomalous charm- $W$ couplings from a number of $C P$-conserving and -violating processes involving the kaon and other flavored hadrons.
\end{abstract}

2009 KAON International Conference

June 09 - 12, 2009

Tsukuba, Japan

*Speaker.

${ }^{\dagger}$ This research was supported in part by NSC and NCTS. 
Current data on low-energy transitions involving the kaon and other flavored hadrons have confirmed the loop-induced flavor-changing neutral current (FCNC) picture of the standard model (SM) and the unitarity of the Cabibbo-Kobayashi-Maskawa (CKM) matrix with three generations [1]. However, there is a growing realization that the present understanding of the dynamics of flavor is incomplete and that physics beyond the SM may be detected in the near future. The continuing study of FCNC processes with increased precision will, therefore, play a crucial role in the search for new physics.

In many scenarios of new physics, the new particles are heavier than their SM counterparts and their effects can be described by an effective low-energy theory. It is possible that the main effect of such new physics is to modify the SM couplings between gauge bosons and certain fermions [2]. The case of anomalous top-quark couplings has been treated before in the literature [3], and it was found that they are stringently constrained by the $b \rightarrow s \gamma$ decay. Interestingly, this mode does not place severe restrictions on anomalous charm-quark couplings because of the relative smallness of the charm mass. Here we present the results of a recent study on the possibility of new physics affecting primarily the charged weak currents involving the charm quark [4].

The effective Lagrangian in the unitary gauge for a general parametrization of anomalous interactions of the $W$ boson with an up-type quark $U_{k}$ and a down-type quark $D_{l}$ can be written as

$$
\mathscr{L}_{U D W}=-\frac{g}{\sqrt{2}} V_{k l} \bar{U}_{k} \gamma^{\mu}\left[\left(1+\kappa_{k l}^{\mathrm{L}}\right) P_{\mathrm{L}}+\kappa_{k l}^{\mathrm{R}} P_{\mathrm{R}}\right] D_{l} W_{\mu}^{+}+\text {H.c. },
$$

where $g$ is the weak coupling constant, the anomalous couplings $\kappa_{k l}^{\mathrm{L}, \mathrm{R}}$ are normalized relative to the usual CKM-matrix elements $V_{k l}$, and $P_{\mathrm{L}, \mathrm{R}}=\frac{1}{2}\left(1 \mp \gamma_{5}\right)$. Thus the SM limit corresponds to $\kappa_{k l}^{\mathrm{L}, \mathrm{R}} \rightarrow 0$. In general, $\kappa_{k l}^{\mathrm{L}, \mathrm{R}}$ are complex and, as such, provide new sources of $C P$ violation.

These new couplings contribute to flavor-changing processes at one-loop level and, therefore, affect loop-generated transitions, such as $K \rightarrow \pi v \bar{v}, K_{L} \rightarrow \ell^{+} \ell^{-}$, and neutral-meson mixing. The relevant loop diagrams are displayed in Fig. $1 .{ }^{1}$ Such processes can be used to place bounds on anomalous couplings in the charm sector. Below we focus on kaon transitions, but will briefly discuss loop-induced, as well as tree-level, transitions involving other flavored hadrons.

Since the effective theory with anomalous couplings is not renormalizable, there are divergent contributions to some of the transitions we consider. Regarding these divergences as contributions to the coefficients of higher-dimension operators in the theory, we handle them by following the common procedure of using dimensional regularization, dropping the resulting pole in four dimensions, and identifying the renormalization scale $\mu$ with the scale $\Lambda$ of the new physics underlying
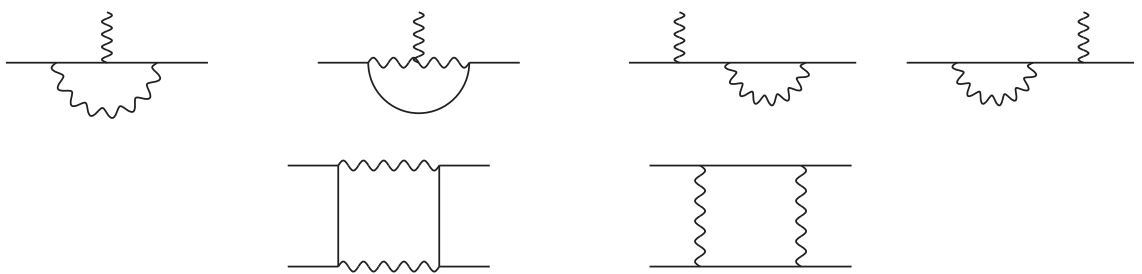

Figure 1: Diagrams contributing to the loop-induced processes discussed in the text. Straight lines (external wavy lines) denote fermions (neutral gauge bosons), and the loops contain $W$ bosons besides fermions.

\footnotetext{
${ }^{1}$ The details of our loop calculation are presented in Ref. [5].
} 
the effective theory. Our results thus contain a logarithmic term of the form $\ln \left(\mu / m_{W}\right)$ in which we set $\mu=\Lambda=1 \mathrm{TeV}$ for definiteness. In addition, we keep in our estimates those finite terms that correspond to contributions from SM quarks in the loops. By adopting this procedure, we trade the possibility of obtaining precise predictions in specific models for order-of-magnitude estimates of the effects of new physics parametrized in a model-independent way.

The first transition we consider is the decay $K^{+} \rightarrow \pi^{+} v \bar{v}$. To quantify the contribution of the anomalous charm couplings to this mode, it is convenient to compare it with the dominant contribution in the SM, which comes from the top loop, and to neglect the masses of the leptons associated with the neutrinos in the new contribution, so that we can work with just one of them. The total amplitude can be written in terms of the dominant SM amplitude as [4]

$$
\begin{gathered}
\mathscr{M}\left(K^{+} \rightarrow \pi^{+} v \bar{v}\right)=(1+\delta) \mathscr{M}_{\mathrm{SM}}\left(K^{+} \rightarrow \pi^{+} v \bar{v}\right), \\
\delta=\frac{V_{c d} V_{c s}^{*}}{V_{t d} V_{t s}^{*}} \frac{\left(\kappa_{c d}^{\mathrm{L}}+\kappa_{c s}^{\mathrm{L} *}\right)\left[-3 \ln \left(\Lambda / m_{W}\right)+4 X_{0}\left(x_{c}\right)\right]}{4 X\left(x_{t}\right)},
\end{gathered}
$$

where we have kept terms to linear order in the $\kappa$ 's, $x_{q}=m_{q}^{2} / m_{W}^{2}, X_{0}\left(x_{t}\right)$ is a loop function, and $X\left(x_{t}\right) \simeq 1.4$ the QCD-corrected value of $X_{0}$ [6]. The SM prediction for the branching ratio is $\mathscr{B}_{\mathrm{SM}}\left(K^{+} \rightarrow \pi^{+} v \bar{v}\right)=(8.5 \pm 0.7) \times 10^{-11}$ [6], to be compared with its experimental value $\mathscr{B}_{\exp }=\left(1.73_{-1.05}^{+1.15}\right) \times 10^{-10}$ [7]. Accordingly, we require $-0.2 \leq \operatorname{Re} \delta \leq 1$, which leads to [4]

$$
-2.5 \times 10^{-4} \leq-\operatorname{Re}\left(\kappa_{c d}^{\mathrm{L}}+\kappa_{c s}^{\mathrm{L}}\right)+0.42 \operatorname{Im}\left(\kappa_{c d}^{\mathrm{L}}-\kappa_{c s}^{\mathrm{L}}\right) \leq 1.3 \times 10^{-3} .
$$

The next decay of interest is $K_{L} \rightarrow \mu^{+} \mu^{-}$. The dominant part of the short-distance contribution to the SM amplitude for this mode is again induced by the top loop. Including the anomalous charm contribution at linear order in $\kappa$, the total short-distance amplitude is [4]

$$
\begin{gathered}
\mathscr{M}_{\mathrm{SD}}\left(K_{L} \rightarrow \mu^{+} \mu^{-}\right)=\left(1+\delta^{\prime}\right) \mathscr{M}_{\mathrm{SM}}^{\mathrm{SD}}\left(K_{L} \rightarrow \mu^{+} \mu^{-}\right), \\
\delta^{\prime}=\frac{\operatorname{Re}\left[V_{c d}^{*} V_{c s}\left(\kappa_{c s}^{\mathrm{L}}+\kappa_{c d}^{\mathrm{L} *}\right)\right]\left[-3 \ln \left(\Lambda / m_{W}\right)+4 Y_{0}\left(x_{c}\right)\right]}{4 \operatorname{Re}\left(V_{t d}^{*} V_{t s}\right) Y\left(x_{t}\right)},
\end{gathered}
$$

where $Y_{0}\left(x_{t}\right)$ is a loop function and $Y\left(x_{t}\right) \simeq 0.95$ its QCD-corrected value [8]. Since the measured branching ratio, $\mathscr{B}\left(K_{L} \rightarrow \mu^{+} \mu^{-}\right)=(6.84 \pm 0.11) \times 10^{-9}$ [1], is almost saturated by the absorptive part of the long-distance contribution, $\mathscr{B}_{\text {abs }}=(6.64 \pm 0.07) \times 10^{-9}$ [9], the difference between them suggests the allowed room for new physics, $\mathscr{B}_{\mathrm{NP}} \lesssim 3.8 \times 10^{-10}$, the upper bound being about one half of the SM short-distance contribution, $\mathscr{B}_{\mathrm{SM}}^{\mathrm{SD}}=(7.9 \pm 1.2) \times 10^{-10}$ [8]. Consequently, we demand $\left|\boldsymbol{\delta}^{\prime}\right| \leq 0.2$, which implies [4]

$$
\left|\operatorname{Re}\left(\kappa_{c s}^{\mathrm{L}}+\kappa_{c d}^{\mathrm{L}}\right)+6 \times 10^{-4} \operatorname{Im}\left(\kappa_{c s}^{\mathrm{L}}-\kappa_{c d}^{\mathrm{L}}\right)\right| \leq 1.5 \times 10^{-4} .
$$

We turn now to constraints from $K-\bar{K}$ mixing. The contribution of the anomalous charm couplings to the matrix element $M_{12}^{K}$ for the mixing is given by [4]

$$
\begin{array}{r}
M_{12}^{K, \kappa}=\frac{G_{\mathrm{F}}^{2} m_{W}^{2}}{24 \pi^{2}} f_{K}^{2} m_{K} V_{c d}^{*} V_{c s}\left[\bar{\eta}^{3} B_{K}\left(\kappa_{c d}^{\mathrm{L} *}+\kappa_{c s}^{\mathrm{L}}\right)\left(-V_{t d}^{*} V_{t s} x_{t} \ln \frac{\Lambda^{2}}{m_{W}^{2}}-\sum_{q} V_{q d}^{*} V_{q s} \mathscr{B}_{1}\left(x_{q}, x_{c}\right)\right)\right. \\
\left.+\frac{\bar{\eta}^{3 / 2} B_{K} m_{K}^{2}}{\left(m_{d}+m_{s}\right)^{2}} \kappa_{c d}^{\mathrm{R} *} \kappa_{c s}^{\mathrm{R}}\left(V_{t d}^{*} V_{t s} x_{t} \ln \frac{\Lambda^{2}}{m_{W}^{2}}+\sum_{q} V_{q d}^{*} V_{q s} \mathscr{B}_{2}\left(x_{q}, x_{c}\right)\right)\right]
\end{array}
$$


where $\bar{\eta}$ is a QCD-correction factor and $\mathscr{B}_{1,2}$ are loop functions, with further details given in Ref. [4]. The $K_{L}-K_{S}$ mass difference $\Delta M_{K}$ is related to $M_{12}^{K}=M_{12}^{K, \mathrm{SM}}+M_{12}^{K, K}$ by $\Delta M_{K}=2 \operatorname{Re} M_{12}^{K}+$ $\Delta M_{K}^{\mathrm{LD}}$, the long-distance term $\Delta M_{K}^{\mathrm{LD}}$ being sizable [10]. Since the LD part has significant uncertainties, we constrain the anomalous couplings by requiring that their contribution to $\Delta M_{K}$ be less than the largest SM SD contribution, which comes from the charm loop [10]. The result is [4]

$$
\left|0.043 \operatorname{Re}\left(\kappa_{c d}^{\mathrm{L}}+\kappa_{c s}^{\mathrm{L}}\right)+0.015 \operatorname{Im}\left(\kappa_{c d}^{\mathrm{L}}-\kappa_{c s}^{\mathrm{L}}\right)-\operatorname{Re}\left(\kappa_{c d}^{\mathrm{R} *} \kappa_{c s}^{\mathrm{R}}\right)+0.28 \operatorname{Im}\left(\kappa_{c d}^{\mathrm{R} *} \kappa_{c s}^{\mathrm{R}}\right)\right| \leq 8.5 \times 10^{-4} .
$$

A complementary constraint on the couplings can be obtained from the $C P$-violation parameter $\varepsilon$. It is related to $M_{12}^{K}$ by $\sqrt{2}|\varepsilon| \simeq\left|\operatorname{Im} M_{12}^{K}\right| / \Delta M_{K}^{\exp }[10]$, where $\Delta M_{K}^{\exp }=(3.483 \pm 0.006) \times$ $10^{-15} \mathrm{GeV}$ [1] and the small term containing the $C P$-violating phase in the $K \rightarrow \pi \pi$ amplitude has been dropped. Since $|\varepsilon|_{\exp }=(2.229 \pm 0.012) \times 10^{-3}[1]$ and $|\varepsilon|_{\mathrm{SM}}=\left(2.06_{-0.53}^{+0.47}\right) \times 10^{-3}$ [11], we require $|\varepsilon|_{\kappa}<0.7 \times 10^{-3}$ for the contribution in Eq. (8). This translates into [4]

$$
\left|0.015 \operatorname{Re}\left(\kappa_{c s}^{\mathrm{L}}+\kappa_{c d}^{\mathrm{L}}\right)+0.043 \operatorname{Im}\left(\kappa_{c s}^{\mathrm{L}}-\kappa_{c d}^{\mathrm{L}}\right)-0.28 \operatorname{Re}\left(\kappa_{c d}^{\mathrm{R} *} \kappa_{c s}^{\mathrm{R}}\right)-\operatorname{Im}\left(\kappa_{c d}^{\mathrm{R} *} \kappa_{c s}^{\mathrm{R}}\right)\right| \leq 2.5 \times 10^{-6}
$$

The anomalous charm couplings also contribute via gluonic dipole operators to $\varepsilon$ and the $C P$ violation parameter $\varepsilon^{\prime}$ in kaon decay, as well as to $C P$ violation in hyperon nonleptonic decays [12]. These operators are generated by the upper diagrams in Fig. 1. The flavor-conserving counterparts of the gluonic (and electromagnetic) dipole operators contribute to the electric dipole moment of the neutron [13]. From the corresponding experimental data, we extract [4]

$$
\left|\operatorname{Im} \kappa_{c d}^{\mathrm{R}}\right| \lesssim 2 \times 10^{-3}, \quad\left|\operatorname{Im} \kappa_{c s}^{\mathrm{R}}\right| \lesssim 2 \times 10^{-3} .
$$

Now we briefly discuss constraints from loop-induced transitions involving $B_{d, s}$ mesons. As in the kaon mixing case, the anomalous charm couplings affect $B_{d}$ mixing. The matrix element $M_{12}^{d}=M_{12}^{d, \mathrm{SM}}+M_{12}^{d, \kappa}$ for the mixing is related to the mass difference $\Delta M_{d}=2\left|M_{12}^{d}\right|$ between the heavy and light mass-eigenstates [10]. The measured and SM numbers $\Delta M_{d}^{\exp }=(0.507 \pm$ $0.005) \mathrm{ps}^{-1}[1]$ and $\Delta M_{d}^{\mathrm{SM}}=\left(0.563_{-0.076}^{+0.068}\right) \mathrm{ps}^{-1}[11]$ are then related by $\Delta M_{d}^{\mathrm{exp}}=\Delta M_{d}^{\mathrm{SM}}\left|1+\delta_{d}\right|$ and $\delta_{d}=M_{12}^{d, \kappa} / M_{12}^{d, \mathrm{SM}}$. Accordingly, we impose $-0.2 \leq \operatorname{Re} \delta_{d} \leq+0.02$, which leads to [4]

$$
-0.031 \leq \operatorname{Re}\left(\kappa_{c b}^{\mathrm{L}}+\kappa_{c d}^{\mathrm{L}}\right)+0.4 \operatorname{Im}\left(\kappa_{c b}^{\mathrm{L}}-\kappa_{c d}^{\mathrm{L}}\right) \leq 0.003 .
$$

An additional constraint can be determined from the measurement of $\beta$ parametrizing mixinginduced $C P$ violation in $B \rightarrow J / \psi K$. The anomalous couplings enter via both the mixing and decay amplitudes. Upon comparing the effective experimental value $2 \beta^{\text {eff }}=0.717 \pm 0.033$ [14] with the SM prediction $2 \beta^{\mathrm{SM}}=0.753_{-0.028}^{+0.032}$ [11], we obtain [4]

$$
-1.5 \times 10^{-3} \leq 0.4 \operatorname{Re}\left(\kappa_{c b}^{\mathrm{L}}+\kappa_{c d}^{\mathrm{L}}\right)-0.69 \operatorname{Im} \kappa_{c b}^{\mathrm{L}}+\operatorname{Im} \kappa_{c d}^{\mathrm{L}}-0.31 \operatorname{Im} \kappa_{c s}^{\mathrm{L}} \leq 0.012 .
$$

There are analogous constraints from the $B_{s}$ sector. Using the experimental and SM values $\Delta M_{s}^{\exp }=(17.77 \pm 0.12) \mathrm{ps}^{-1}[1]$ and $\Delta M_{s}^{\mathrm{SM}}=\left(17.6_{-1.8}^{+1.7}\right) \mathrm{ps}^{-1}[11]$, we arrive at [4]

$$
-0.014 \leq \operatorname{Re}\left(\kappa_{c s}^{\mathrm{L}}+\kappa_{c b}^{\mathrm{L}}\right)+0.018 \operatorname{Im}\left(\kappa_{c s}^{\mathrm{L}}-\kappa_{c b}^{\mathrm{L}}\right) \leq 0.015 .
$$

A complementary constraint is provided by the parameter $\beta_{s}$ in $B_{s}$ decay, analogously to $\beta$ in $B_{d}$ decay. In this case, the mode of interest is $\bar{B}_{s}^{0} \rightarrow J / \psi \phi$, which proceeds from the same 
$b \rightarrow s c \bar{c}$ transition as $\bar{B}_{d}^{0} \rightarrow J / \psi \bar{K}$. The current disagreement between the SM number $2 \beta_{s}^{\mathrm{SM}}=$ $0.03614_{-0.00162}^{+0.00172}[11]$ and the measured value $2 \beta_{s}^{\text {eff }}=2 \beta_{\psi \phi}^{\text {eff }}=0.77_{-0.29}^{+0.37}$ or $2.36_{-0.37}^{+0.29}$ [14] can be used to extract [4]

$$
-0.09 \leq 0.026 \operatorname{Re}\left(\kappa_{c b}^{\mathrm{L}}+\kappa_{c s}^{\mathrm{L}}\right)+\operatorname{Im}\left(\kappa_{c b}^{\mathrm{L}}-\kappa_{c s}^{\mathrm{L}}\right) \leq 7 \times 10^{-4} .
$$

The anomalous charm couplings affect not only loop-induced amplitudes, but also tree-level ones. The couplings contribute at tree level to $D \rightarrow \ell v$ and $D_{s} \rightarrow \ell v$. Existing experimental and theoretical values of the decay constant $f_{D}$ are consistent with each other, but those of $f_{D_{s}}$ disagree at the 2-sigma level [1, 15]. This information leads us to find [4]

$$
\left|\operatorname{Re}\left(\kappa_{c d}^{\mathrm{L}}-\kappa_{c d}^{\mathrm{R}}\right)\right| \leq 0.04, \quad 0 \leq \operatorname{Re}\left(\kappa_{c s}^{\mathrm{L}}-\kappa_{c s}^{\mathrm{R}}\right) \leq 0.1 .
$$

Another process affected by the anomalous charm couplings at tree level is the semileptonic decay $b \rightarrow c e^{-} \bar{v}_{e}$. Consequently, they would pollute the extraction of $V_{c b}$. From the experimental results on the exclusive modes $\bar{B} \rightarrow\left(D, D^{*}\right) e \bar{v}_{e}$ and the inclusive one [1], we arrive at [4]

$$
-0.13 \leq \operatorname{Re} \kappa_{c b}^{\mathrm{R}} \leq 0 .
$$

Lastly, constraints on the couplings can be obtained from comparing the measurements of the $\mathrm{CP}$ violation parameter $\beta$ in $B \rightarrow J / \psi K$ and $B \rightarrow \eta_{c} K$. The SM predicts the same $\sin (2 \beta)$ for the two processes, whereas the present data for its effective values are $\sin \left(2 \beta_{\psi K}^{\text {eff }}\right)=0.657 \pm 0.025$ and $\sin \left(2 \beta_{\eta_{c} K}^{\text {eff }}\right)=0.93 \pm 0.17$ [14], which disagree at the 1.5 -sigma level. This could be due to tree-level effects of the anomalous couplings, which would imply [4]

$$
-5 \times 10^{-4} \leq \operatorname{Im}\left(\kappa_{c b}^{\mathrm{R}}+\kappa_{c s}^{\mathrm{R}}\right) \leq 0.04 .
$$

In conclusion, we have explored the phenomenological consequences of anomalous $W$-boson couplings to the charm quark in a comprehensive way. Kaon processes can be seen to have yielded some of the strongest bounds on the couplings. In order to gain more insight into the constraints obtained above, we have derived from them the ranges corresponding to taking only one anomalous coupling at a time to be nonzero (and only for the cases of a purely real or a purely imaginary coupling). They are collected in Table $1 .^{2}$ This table shows that the resulting constraints on the

\begin{tabular}{|c||c|}
\hline \hline $0 \leq \operatorname{Re} \kappa_{c d}^{\mathrm{L}} \leq 1.5 \times 10^{-4}$ & $\left(\operatorname{Im} \kappa_{c d}^{\mathrm{L}}=0\right)$ \\
$0 \leq \operatorname{Re} \kappa_{c s}^{\mathrm{L}} \leq 1.5 \times 10^{-4}$ & $-6 \times 10^{-5} \leq \operatorname{Im} \kappa_{c s}^{\mathrm{L}} \leq 6 \times 10^{-5}$ \\
$-4 \times 10^{-3} \leq \operatorname{Re} \kappa_{c b}^{\mathrm{L}} \leq 3 \times 10^{-3}$ & $-0.02 \leq \operatorname{Im} \kappa_{c b}^{\mathrm{L}} \leq 7 \times 10^{-4}$ \\
$-0.04 \leq \operatorname{Re} \kappa_{c d}^{\mathrm{R}} \leq 0.04$ & $-2 \times 10^{-3} \leq \operatorname{Im} \kappa_{c d}^{\mathrm{R}} \leq 2 \times 10^{-3}$ \\
$-0.1 \leq \operatorname{Re} \kappa_{c s}^{\mathrm{R}} \leq 0$ & $-5 \times 10^{-4} \leq \operatorname{Im} \kappa_{c s}^{\mathrm{R}} \leq 2 \times 10^{-3}$ \\
$-0.13 \leq \operatorname{Re} \kappa_{c b}^{\mathrm{R}} \leq 0$ & $-5 \times 10^{-4} \leq \operatorname{Im} \kappa_{c b}^{\mathrm{R}} \leq 0.04$ \\
\hline \hline
\end{tabular}

Table 1: Constraints on each of the anomalous charm couplings.

\footnotetext{
${ }^{2}$ In determining the left-handed entries in this table, we have taken into account the fact that only two relative phases among the three left-handed charm- $W$ couplings are physical [4] and accordingly chosen $\phi_{c d}^{\mathrm{L}}=0$.
} 
anomalous charm couplings are, perhaps surprisingly, comparable or tighter than existing constraints on anomalous $W$-boson couplings to the top quark. The table also shows that, in general, the left-handed couplings are much more constrained than the right-handed couplings. Similarly, the imaginary part of the couplings is more tightly constrained than the corresponding real part. The biggest deviations allowed by current data appear in the real part of the right-handed couplings, which can be as large as $10 \%$ of the corresponding SM couplings. Finally, our study can also serve as a guide as to which future measurements provide the most sensitive tests for new physics that can be parametrized with anomalous charm- $W$ couplings.

\section{References}

[1] C. Amsler et al. [Particle Data Group], Phys. Lett. B 667, 1 (2008).

[2] R.D. Peccei and X. Zhang, Nucl. Phys. B 337, 269 (1990); R.D. Peccei et al., ibid. 349, 305 (1991).

[3] K. Fujikawa and A. Yamada, Phys. Rev. D 49, 5890 (1994); D.O. Carlson et al., Phys. Lett. B 337, 145 (1994); A. Abd El-Hady and G. Valencia, ibid. 414, 173 (1997); F. Larios et al., ibid. 457, 334 (1999); G. Burdman et al., Phys. Rev. D 61, 114016 (2000); B. Grzadkowski and M. Misiak, ibid. 78, 077501 (2008); J.P. Lee and K.Y. Lee, ibid. 78, 056004 (2008).

[4] X.G. He, J. Tandean, and G. Valencia, Phys. Rev. D 80, 035021 (2009) [arXiv:0904.2301 [hep-ph]].

[5] X.G. He, J. Tandean, and G. Valencia, Eur. Phys. J. C 64, 681 (2009) [arXiv:0909.3638 [hep-ph]].

[6] A.J. Buras et al., JHEP 0611, 002 (2006); F. Mescia and C. Smith, Phys. Rev. D 76, 034017 (2007); A.J. Buras et al., Rev. Mod. Phys. 80, 965 (2008); J. Brod and M. Gorbahn, Phys. Rev. D 78, 034006 (2008).

[7] A.V. Artamonov et al. [E949 Collaboration], Phys. Rev. Lett. 101, 191802 (2008).

[8] M. Gorbahn and U. Haisch, Phys. Rev. Lett. 97, 122002 (2006).

[9] L. Littenberg and G. Valencia, in Ref. [1].

[10] G. Buchalla, A.J. Buras, and M.E. Lautenbacher, Rev. Mod. Phys. 68, 1125 (1996).

[11] CKMfitter, http://ckmfitter.in2p3.fr.

[12] J.F. Donoghue and B.R. Holstein, Phys. Rev. D 32, 1152 (1985); J.F. Donoghue et al., ibid. 34, 833 (1986); X.G. He and G. Valencia, ibid. 52, 5257 (1995); A.J. Buras et al., Nucl. Phys. B 566, 3 (2000); X.G. He et al., Phys. Rev. D 61, 071701 (2000); J. Tandean, ibid. 69, 076008 (2004).

[13] X.G. He et al., Int. J. Mod. Phys. A 4, 5011 (1989) [Erratum-ibid. A 6, 1063 (1991)]; Phys. Lett. B 254, 231 (1991); C. Dib et al., J. Phys. G 32, 547 (2006).

[14] Heavy Flavor Averaging Group, http://www.slac.stanford.edu/xorg/hfag.

[15] S. Stone, arXiv:0806.3921 [hep-ex]; B.A. Dobrescu and A.S. Kronfeld, Phys. Rev. Lett. 100, 241802 (2008); A.S. Kronfeld, PoS LATTICE2008, 282 (2008); J. P. Alexander et al. [CLEO Collaboration], Phys. Rev. D 79, 052001 (2009). 